\title{
LES SAISONS DE L'ÂME CHEZ ANNA DE NOAILLES: NATURE ET INSPIRATION
}

\author{
Gilmara Viviane Castor de Andrade ${ }^{1}$ \\ Karina Chianca ${ }^{2}$
}

RÉSUMÉ: Lorsque nous étudions la poésie d'Anna de Noailles, nous sommes d'abord frappés par l'environnement historique et social dans lequel elle s'inscrit. La première impression dégagée est qu'elle pourrait se placer hors contexte par rapport à cette période. Anna de Noailles a préféré gardé sur ses poèmes des thèmes simples s'inspirant de la nature et des mythes grecs, s'éloignant de la thématique de la modernité, telle que la modernisation des villes, l'électricité et la vélocité. En effet, il s'agit de la bouleversante période de la fin du XIXème siècle et du début du XXème. Ses poèmes gardent en soi un moi lyrique qu'on pourrait le placer dans le mouvement romantique, aussi nous pouvons observer chez Noailles des thèmes liés à la nature et aux dieux grecs. Ce qui va à contre-courant de la poésie moderne qui naît en France à la fin du XIXème siècle.

MOTS-CLÉS: Nature, poésie, inspiration, modernité, lyrisme.

\begin{abstract}
When we study Anna de Noailles' poetry, we are fast concerned by the social and historical context which she is represented. The first idea we have about her, it's that she could be away of the context itself related to that time. Anna de Noailles has preferred to keep his poems on simple themes inspired by nature and Greek myths, away from themes of modernity, such as the modernization of cities and velocity. It deals with the hard end of the 19th century and the beginning of the 20th one. Her poems keep inside a lyric self that could be contextualized into the Romantic Movement. We can also realize in Noailles, subjects connected with the nature and some Greek gods. And it means they go against the idea of the modern poetry which is born in France at the end of the 19th century.
\end{abstract}

KEYWORDS: nature, poetry, inspiration, modernity, lyricism.

\footnotetext{
${ }^{1}$ ANDRADE, Gilmara Viviane Castor de. Mestranda UFRN. E-mail: gilmaravie@yahoo.fr

${ }^{2}$ CHIANCA, Karina, Professora Doutora UFPB. E-mail: kachianca@yahoo.fr
} 


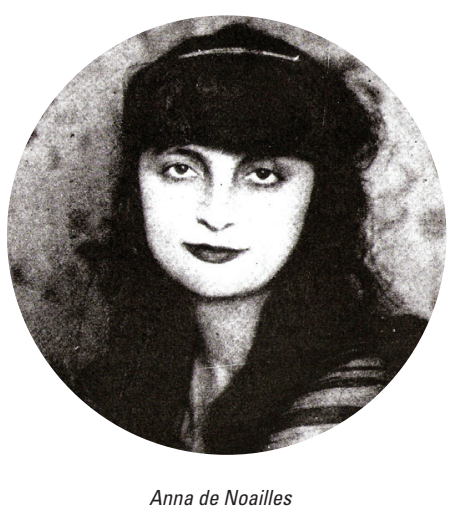

\section{INTRODUCTION}

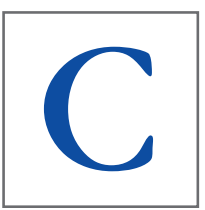

Noailles.

Lorsque nous avons commencé notre recherche sur cette poète, nous nous sommes immédiatement rendues compte du manque d'études critiques sur ses œuvres. Cela nous a apporté quelques difficultés en ce qui concerne le développement de notre étude.

Malgré la grandiosité de la poésie noaillienne, elle est peu étudiée en France. Quant au Brésil d'après ce que nous avons recherché, il n'y existe pas d'études sur cette poète. Nous espérons apporter une contribution pour l'académie avec ce bref article.

Notre appui critique est essentiellement basé, en ce qui concerne la poésie d'Anna de Noailles, sur le livre d'Angela Bargenda, critique littéraire des œuvres noailliennes. C'est pour cette raison que nous la citerons tout au long de notre recherche. Une autre difficulté que nous avons ressentie, est le fait que le recueil L’Ombre des Jours d'Anna de Noailles soit épuisé, cela nous a amenées à le chercher sur une bibliothèque électronique en France.

\section{LE CONTEXTE HISTORIQUE}

Lorsque nous étudions la poésie d'Anna de Noailles, nous sommes d'abord frappés par l'environnement historique et social dans lequel elle s'inscrit. La première impression dégagée est qu'elle pourrait se placer hors contexte par rapport à cette période d'innovation dans le champ de la littérature et des arts. Anna de Noailles a préféré garder sur ses poèmes des thèmes simples s'inspirant de la nature et des mythes grecs, s'éloignant de la thématique de la modernité, telle que 
la modernisation des villes, l'électricité et la vélocité. En effet, il s'agit de la bouleversante période de la fin du XIXème siècle et du début du XXème (BERCOT, 2000, p. 285). À cette époque, la France passait par une crise morale et de désenchantement du monde moderne. Les conséquences catastrophiques de la révolution industrielle, la Restauration, la guerre Franco-prussienne et la première Grande Guerre ont marqué les mouvements artistiques en France. À la fin du XIXème siècle, le poète se sent incompris par la société qui l'entoure, c'est le cas de Baudelaire, de Verlaine et de Rimbaud. ${ }^{3} \mathrm{Il}$ met en question ce qui va devenir la société moderne, et c'est dans ce monde d'incertitude que l'inspiration poétique prend sa source. La confrontation avec la dure réalité des conflits, tant au plan moral, que social, pousse les poètes à écrire sur le réel, sur les expériences et sur les sensations vécues.

La question que les poètes se posaient à l'époque était : pourquoi continuer à écrire de la poésie? (BERCOT, 2000, p. 285) Il fallait trouver sa place dans le chaos du monde moderne. Nous avons comme exemple du début du XXème siècle la poésie d'Apollinaire avec Alcools ou Calligrammes et Blaise Cendrars avec son célèbre poème La prose du Transsibérien, tous les deux ont rompu avec la forme traditionnelle. ${ }^{4}$ Au XIXème siècle, quelques auteurs se sont refugiés dans les songes pour fuir la réalité, transformant un objet réel en un objet imaginaire, dessiné selon le regard de l'artiste. Les auteurs du XXème siècle vont plutôt tenir un tout autre langage: c'est l'adéquation de l'art au monde moderne qui importe.

La poésie d'Anna de Noailles est née dans ce contexte, et en opposition à cette conception de la vie et de l'art. Son désenchantement pour la vie n'est pas sur le plan social, mais sur le plan amoureux. Sa poésie, d'une très forte tradition lyrique et romantique, va à contrecourant du mouvement symboliste (fin du XIXème siècle) et surréaliste (début du XXème siècle) que nous trouvons en France. Ces deux mouvements littéraires annonçaient un poète retourné à soi-même, cherchant à exprimer le plus profonde de l'âme, mais à la différence de notre poète, ces poètes suggèrent plus que ne définissent. Par contre Anna de Noailles cherche à travers la langue exprimer ses sensations la plus claire possible. Selon Angela Bargenda «Contrairement au symbolisme, où les notions abstraites et irréelles sont largement favorisées, Anna de Noailles opte pour un vocabulaire concret et tangible» (BARGENDA, 1995, p. 63).

Michel Pringent dit à ce sujet que "Le poète moderne vise à exprimer une intime altérité plutôt que son identité, cultive la distance plus que la fidélité au réel, l'écart, non la filiation vis-à-vis de la tradition" (PRINGENT, 2006, p. 837).

Alors que ses contemporains privilégiaient la rupture de la forme et la destruction de la rime, Anna de Noailles reste fidèle à ces caractéristiques de la tradition poétique. Pour citer encore Michel Pringent; "la modernité tend au contraire à privilégier les ruptures, au point de faire disparaitre toute structure reconnaissable" (PRINGENT, 2006, p. 837). Les mouvements littéraires de l'époque cherchaient à affirmer une nouvelle identité dans le domaine poétique, et à s'éloigner de la tradition. Pour le mouvement futuriste du début du XXème siècle, la beauté du monde moderne était une autre, marquée par le progrès et par la vitesse (BERCOT, 2000, p. 293). Ainsi, la poésie

\footnotetext{
${ }^{3}$ Poussés par le désenchantement de la société dans laquelle ils vivent, ces poètes ont puisé dans la tradition lyrique leurs chants poétiques, les cris du cœur et l'angoisse d'exister afin d'apaiser leurs âmes.

${ }^{4}$ Les poètes jouaient avec la forme, quelques-uns de leurs poèmes sont en vers libres et ils ont même abolit toute ponctuation.
} 
change sa thématique, ce n'est plus le goût pour la nature, telle qu'elle était peinte au XIXème siècle, qui est en évidence dans ce mouvement, mais les inquiétudes du monde moderne. Nous devons citer ici des poètes tels que Victor Hugo et Baudelaire qui, comme l'affirme Martine Bercot (2000, p. 293), malgré leur appartenance à des courants du XIXème siècle, n'ignoraient pas les conflits sociaux de leur époque. L'auteur souligne le fait que ces deux poètes s'exprimaient d'une façon allégorique. Toujours selon l'auteur, la poésie du XIXème siècle était, d'une certaine façon, comme absente du monde réel.

\section{ANNA DE NOAILLES ET SON MILIEU SOCIAL}

Anna de Noailles est née en 1876 à Paris, d'un père roumain et d'une mère crétoise, pianiste célèbre. Du côté paternel, son père, le prince Grégoire de Bassaraba-Brancovan, descendait de la dynastie moldave des Bassaraba. Toutefois, l'ascendance maternelle de son père était grecque. À notre avis, cette origine grecque a une forte influence dans l'œuvre d'Anna de Noailles.

Sa carrière littéraire débute en 1889, après son mariage avec le comte Mathieu de Noailles. Ses premières publications ont été dans la Revue de Paris et dans la Revue des Deux Mondes. En 1901, elle publie le recueil Le Cour innombrable, chez Calman-Lévy et en 1902, L’Ombre des Jours, chez le même éditeur. Anna de Noailles a aussi publié quelques romans, mais son succès comme écrivain est dû à la poésie.

Anna de Noailles a été une figure connue à l'époque, Aristide Briand, Jean Jaurès, Paul Valéry, André Gide, Jean Cocteau et Maurice Barrès faisaient partie de son cercle d'amitié, les trois derniers ont maintenu une correspondance avec elle. La poète était célèbre dans son temps, elle a reçu le Prix Fémina et la cravate de Commandeur de la Légion d'Honneur. Malheureusement, son œuvre est peu connue par les contemporains. On la voit plus comme une figure mondaine qu'artistique.

L'acte d'écrire pour Anna de Noailles est plus qu'un exercice de langage, il est thérapeutique ${ }^{5}$ et constitue aussi une manière d'être toujours présent dans les générations futures. Nous avons observé chez la poète cette préoccupation de l'universalité:

J'écris pour que le jour où je ne serais plus

On sache comme l'air et le désir m'ont plu,

Et que mon livre porte à la foule future

Comme j’aimais la vie et l'heureuse Nature

(NOAILLES, 1902, p. 169).

Elle avait toujours ce désir d'écrire, et ne plus le faire serait épouvantable, comme elle l'a déclaré: "Ne plus rien écrire est une épouvante que touché à la folie [...] d'un agissement sans résultants, d'une fin sans avenir" (BARGENDA, 1995, p. 69) ${ }^{6}$. Nous pouvons remarquer dans sa déclaration la conscience qu'elle avait du rôle du poète: il est celui qui doit contribuer à l'avenir de

\footnotetext{
${ }^{5}$ Selon l'ancienne tradition lyrique le chant poétique est delivrance, une élévation de l'homme vers le divin.

${ }^{6}$ Cette déclaration d'Anna de Noailles est citée dans le livre de Bargenda.
} 
la société et partage avec elle les expériences vécues, ainsi que les sensations et les désirs. Quant à sa conception de l'inspiration poétique, Anna de Noailles liait l'acte créateur au divin.

Si je faisais des corrections, je ne reconnaîtrais pas mes poèmes, ils n'évoqueront plus pour moi les moments de l'inspiration, ces minutes dont le poète doit dire avec modestie: non sa propre force, mais par celle que lui communiquait un Dieu (BARGENDA, 1995, p. 1).?

Contrairement à ce que pensait Rimbaud, la création poétique n'était pas le résultat d'un état de délire qui ne durait qu'un espace de temps. Paul Valéry reconnaissait qu'il existait un «état poétique» qui s’installait, se développait, et se désagrégeait en nous (BRUNEL, 2003, p. 90). Pour Noailles, la poésie est libération de son paysage intérieur: «Je ne fais que raconter ce que l’univers à travers moi, porte sans cesse vers mon cœur.»

Dans la poésie d'Anna de Noailles, il existe une tension entre l'ordre et le désordre, peut-être, par l'influence du lyrisme grec. Le "chant" de la poète est désordre et contemplation, errance et demeure (MAULPOIX, 2000, p. 296). Cette constante tension des cordes mène le poète à créer, toujours inspiré par les muses conciliatrices ${ }^{8}$ des contraires. Chez Anna de Noailles, cet instant inspirateur passe par deux moments: le moment de contemplation de la vie et des impressions présentes dans son esprit, pour qu'ensuite ces images gravées dans son âme surgissent en création.

\section{MORTS ET RENAISSANCES}

Nous avons pu remarquer dans les poèmes noailliens un «moi» lyrique cyclothymique, dans la mesure où nous trouvons un fort désir de plénitude, d'expérimenter à tout et à toutes les sensations, pour ensuite désirer la mort, qu'elle soit physique ou poétique. Son poème «Renaissance» nous montre cette aspiration à renaitre après la peine.

\footnotetext{
Je sais que tu tenais à garder prudemment

Cette émouvante peine au plus secret de toi, Mais mon âme, la vigne a rebodé les toits Et l'abeille a repris son bel amusement (NOAILLES, 1902, p. 55)
}

Dans ce poème, le «moi» lyrique est en dialogue avec lui-même, c'est la voix intérieure qui essaie de dissuader l'âme de sa torpeur en montrant la nature qui est renouvelée. À l'image de celle-ci, l'âme doit renaître chaque été, vu son caractère cyclique. Aussi, cette idée de renaissance est renforcée par trois verbes de la 3ème et de la 4ème strophe, les verbes «reprendre», «retourner»

\footnotetext{
${ }^{7}$ Il s'agit ici d'un commentaire d'Anna de Noailles qui porte sur l'« Enquête sur la poésie contemporaine », transcrite dans le livre d'Angela Bargenda.

${ }^{8}$ Les muses inspirent en même temps qu'elles peuvent mener aussi au désespoir.
} 
et «recommencer». Le choix nous montre le passage du temps. Ce temps qui passe et qui apporte toujours avec lui la mort poétique du «moi» lyrique aura une très forte influence dans la poésie d'Anna de Noailles. L'arrivée de l'été emmène la saison de l'amour et des passions, toutefois le «moi» lyrique sait que tout est passager et que la peine ne tardera pas. L'été apporte la joie éphémère et pour cette raison, le sujet ne peut qu'en sortir blessé.

Après avoir remarqué ce temps cyclique dans les poèmes de la poète, nous pouvons faire un lien avec le mythe d'Éros. Éros représente une force d'attraction qui devient nécessaire à la reproduction. En effet, il assure à la fois la cohésion et la pérennité de l'univers dans la cosmogonie hésiodique (BRUNEL, 1988, p. 564-573). Selon le Dictionnaire des mythes littéraires, le pouvoir en excès d'Éros peut détruire, ainsi il faut qu'il y ait un équilibre de la force de l'Amour'. Si nous faisons un rapport avec les périodes de hauts et de bas du «moi» lyrique, nous dirions que cet aspect cyclothymique est nécessaire pour maintenir l'harmonie. Peut-être qu'Anna de Noailles était consciente de cela, le dernier vers du poème Renaissance résume bien ce que nous avons dit. «Puisqu'il faut bien qu'un peu de bonheur recommence...» Ce vers se termine par des points de suspension, suggérant ainsi un caractère mouvant du temps.

Si l'été blesse, le «moi» lyrique retrouvera l'apaisement auprès de l'automne et de l'hiver. Ceux-ci seront les baumes pour guérir les plaies. Dans ces vers du poème «Le Répit», nous pouvons observer le pouvoir consolateur de l'hiver:

O rude et consolant hiver, hiver de neige.

Hiver sans volupté, sans chants et sans odeur,

O saisons sans semailles et sans ferment, protège

L'appesantissement étroit et las du cœur.

Mais tant que sera si prudente la vie

J'entendrai s'apprêter dans les jardins du Temps

Les flèches de soleil, de désir d'envie

Dont l'été blessera mon cœur tendre et flottant...

(NOAILLES, 1902, p. 53)

Ce poème est une prière du «moi» lyrique à l'hiver. Mais nous savons à l'avance que, comme toujours, cette paix ne durera pas. Le premier vers nous montre le côté contradictoire de la saison froide; l'hiver est rude, cependant consolateur. Comme nous l'avons déjà dit, il représente le calme mais aussi le manque d'attraits, d'abondance, de sensations, de sens et de plaisirs, si chers aux mois de l'été. Les sensations qui font éveiller le désir sont mortes, mais le poète sait que le Temps ne s'arrête pas.

La dernière strophe annonce la proximité de l'été. L'instabilité du désir du «moi» lyrique le transforme en une proie facile pour les attractifs de la saison chaude. Les rayons de soleil ici sont transformés en flèches qui blesseront le cœur tendre et flottant du poète. Cette blessure dans un

\footnotetext{
${ }^{9}$ Selon le dictionnaire, Éros est une des trois entités primordiales qui préexistent à la formation de l'univers: l'Abîme (Chaos), la Terre (Gaïa) et l'Amour (Éros). Ils sont issus de cette triade, le Cosmos et les dieux, «Eros joue un rôle particulier, puisque sans engendrer lui-même, il représente une tierce force, une force d'attraction qui devient nécessaire à la reproduction".
} 
premier moment est bénéfique, puisqu'elle apportera un peu de bonheur et de plaisir au cœur. Mais en même temps, le soleil qui blesse apportera l'inquiétude, la tentation et la folie. Nous constatons une contradiction entre la tendresse qui marque le «moi» lyrique avant l'arrivée de la chaleur et la sensualité des images qui suivent.

$\mathrm{V}$

C'est déjà sourdement sous l'herbe et dans les bois

L'impétueux réveil des dieux chauds et vivaces

Qui ramènent, noués ensemble, les trois mois

D'impatient plaisir, de tumulte et d'audace

VI

Alors chez les humains, le sage, l'orgueilleux, Ceux qui goûtaient le plus la science et le livre Viendront imprudemment vers l'été périlleux Et pleureront d'espoir et du désir de vivre.

IX

Le subtil dieu d'erreur et de tentation

Qui vient troubler le goût de l'heure familière, Qui mène la colère et la crispation

Et qui s'enroule autour du désir comme un lierre.

(NOAILLES, 1902, p. 47)

L'adverbe «déjà» marque précisément l'arrivée de l'été, qui comme la poète souligne à la IXème strophe, «il s'enroule autour du désir comme un lierre». Il est donc difficile de lui échapper, parce qu'il nous séduit et nous fait désirer une certaine liberté de sensations. Nous avons observé qu'il y a ici une gradation dans le désir du «moi» lyrique, et que cette gradation coïncide justement avec les trois mois de l'été: «Qui ramènent, noués ensemble, les trois mois/D'impatient plaisir, de tumulte et d'audace.» L'été nous ramène le dieu d'erreur et de tentation, à savoir Dionysos.

Nous sommes encore une fois en présence de la tradition grecque et des dieux qui sont des protagonistes dans la poésie noaillienne. Apollon, Éros et Dionysos, sans parler d'Orphée dans l'expression même du lyrisme. Ils sont mis en scène dans les poèmes et partagent les aventures, les peines, les plaisirs, les folies des êtres humains. Dionysos réapparaît en été pour ses fêtes arrosées par le vin, par l'ivresse et par les cris, le tumulte et à la fin, les peines de cœur. Il marque aussi l'errance dans l'espace et dans le temps. Et après Dionysos, l'hiver prend place, accompagné par le calme mais aussi par la solitude. Mais dans la poésie noaillienne, il s'agit d'une souffrance féminine.

Dans le poème «Les plaintes d'Ariane» (BRUNEL, 1988, p. 160) ${ }^{10}$, ce personnage mythique

\footnotetext{
${ }^{10}$ Ariane était l'amante de Thésée, c'est elle qui l'a aidé à sortir du labyrinthe pour ensuite être abandonnée par celui-ci. Une des versions du mythe affirme qu'Ariane a été l'épouse de Dionysos. Elle devient immortelle et reçoit de lui une constellation, la constellation d'Ariane.
} 
représente la voix lyrique de la souffrance féminine. Contrairement à Orphée, c'est elle qui subi la douleur de la perte. Le terme «plainte» peut être associé à des gémissements, à des cris et nous pouvons aussi l'associer à un chant de mort comme l'élégie ${ }^{11}$. Anna de Noailles prête sa voix à Ariane qui, comme prêtresse, fait résonner son chant.

Le vent qui fait tomber les prunes

Les coings verts,

Qui fait vaciller la lune,

Le vent qui mène la mer,

Le vent qui rompt et qui saccage,

Le vent froid

Qu'il vienne et qu'il fasse rage

Sur mon cœur en désarroi!

(NOAILLES, 1902, p. 107)

Nous pouvons observer que le vent sera traité suivant un ordre croissant, qui augmente et arrive jusqu'à la tempête: «Ah quelle vienne la tempête.» L'évocation du vent commence par celui qui est dispensateur de bienfaits et se termine par ce qui détruit. Les images de la «violence» de la nature, exprime le trouble moral du «moi». Le nom «désarroi» vient renforcer et affirmer ce désordre de l'âme qui se trouve perdue comme dans un labyrinthe. L'amant d'Ariane est sorti du labyrinthe, mais elle est condamnée à y rester.

Ariane, ainsi qu'Orphée, est celle qui souffre pour arriver à la divinisation. Elle est celle qui aime et qui sera par la suite abandonnée, mais l'abandon signifie l'immortalité. Or, Dionysos lui accorde cette immortalité tant désirée, attendri par sa souffrance après l'abandon de Thésée, selon une des versions répandues du mythe.

Quant à la forme du poème, il est composé par des vers heptasyllabes et trissyllabes, ces derniers sont un peu décalés, peut-être pour donner la forme du vent qui souffle violemment. Nous y trouvons aussi des vers octosyllabes.

À notre avis, ce poème est celui qui est le plus proche du symbolisme. Nous y observons toute une sonorité et aussi les sensations visuelles qui viennent s'ajouter pour souligner l'impression de passage du vent. Quand la poète commence par énumérer «Le vent qui fait tomber le prunes/ Les coings verts» [...], nous avons la sensation d'un tourbillon où le «moi» lyrique se trouve perdu. Aussi, les reprises du dernier vers donnent l'image d'un va et vient qui nous montre ce trouble psychologique du «moi» lyrique.

Jusqu'ici nous avons observé chez Noailles des poèmes où apparait la mort poétique, une mort qui est toujours liée aux saisons. Cependant, nous avons pu constater dans quelques poèmes des allusions à la mort physique.

O Mort de t'avoir crainte un jour, je me repens,

O fille de Cybèle auguste et du dieu Pan

${ }^{11}$ Selon le dictionnaire Petit Robert, le mot « plainte » peut être associé à des cris, à des lamentations et à des pleurs. 
Dont les bras on porté la terre et le feuillage, Toi, divine, par qui le cœur est enfin sage,

Que faisais-je quand triste aux approches du soir

Je me cachais de toi et craignais de te voir...

Pourtant pour nous si las, si vains, pour nous qui sommes

Toujours blessés de joie ou d'ennuis, pauvres hommes,

Qui mieux que toi connais le baume et le secret,

Le breuvage, le lit, le vent limpide et frais,

La bonne et harmonieuse et longue sauvegarde...

(NOAILLES, 1902, p. 175)

Dans ce poème la poète avoue son amour à celle-ci, «Je t'aime comme j’ai parfois aimé l'été.», c'est le caractère permanent de la Mort qui attire la poète, "Comme rien, hormis toi, n'est égal ou durable». Dans un premier temps le «moi» lyrique désirait tout vivre et tout voir. Mais lorsqu'il a gouté à toutes les sensations et vécu plusieurs cycles de joies et de peines, sachant que tout a une fin, il aspire à la mort.

La Mort, comme nous pouvons le remarquer dans ce poème, est dispensatrice de bienfaits à l'âme souffrante qui est toujours blessée, soit par la joie, soit par l'ennui. Dans le dernier vers de la deuxième strophe, la poète a la certitude que la Mort représente la fin du désordre et que par conséquent c'est dans celle-ci que se trouve la fin de l'errance. Elle serait ainsi le lieu de la demeure éternelle du «moi» lyrique qui a tant erré et souffert, une demeure associée à la protection et au bien être dans l'éternité, avec le «breuvage», le «lit», le «vent limpide et frais».

Pourtant, il s'agit d'un désir exprimé pour une mort physique, ceci n'est pas tout à fait souhaité en ce qui concerne la mort poétique. Nous avons pu observer que le «moi» lyrique exprime son aspiration de vivre même après sa disparition, car il peut trouver sa demeure éternelle dans l'âme d'autrui:

J'ai dit ce que j'ai vu et ce que j’ai senti,

D'un cœur pour qui le vrai ne fut point trop hardi,

Et j’ai eu cette ardeur, par l'amour intimée,

Pour être après la mort parfois encore aimée,

Et qu'un jeune homme alors lisant ce que j'écris,

Sentant par moi son cœur, ému, troublé, surpris,

Ayant tout oublié des compagnes réelles,

M'accueille dans son âme et me préfère à elles...

(NOAILLES, 1902, p. 169)

\section{CONSIDÉRATIONS FINALES}

La poésie noaillienne garde en elle les thèmes du romantisme français, comme la nature et l'expression d'un lyrisme. Nous avons pu observer que chez Anna de Noailles la nature est 
un thème recourant et que la poète s'en nourri et s'en inspire. Sa poésie traduit son vécu, ses expériences, ses sensations et tous les paysages gardés dans son «âme faunesse», comme Noailles a elle-même avoué.

Il est important de souligner que dans ses poèmes, les mythes grecs ne constituent pas seulement un décor, ils sont aussi la résonance de ses origines crétoises. Chez la poétesse, les mythes sont comme un prolongement de son héritage hellénique. Dans ses poèmes, les dieux et déesses grecs prennent forme et dialoguent, dessinant sur les vers leurs inquiétudes, leurs errances, leurs souffrances et leurs tourments. Les dieux anthropomorphisés partagent ainsi avec l'homme les angoisses de la vie

La nature qui change est à l'image de l'âme du poète puisqu'elle souffre, mais elle sait aussi que tout est cyclique et que la joie vient après la peine. La poésie d'Anna de Noailles nous a aussi montré son lien avec les saisons, que dans son cas, sont associées au psychologique. Le passage du temps marque bien ces «saisons psychologiques». L'automne et l'hiver sont présentés comme le temps de la sagesse et de la guérison pour une nouvelle renaissance après la peine. Noailles se sert aussi de la nature pour aborder le thème de la mort qui peut être aussi bien poétique que physique. En d'autres termes, la nature et l'âme de la poète ne forment qu'une seule chose, les deux conduisant toujours à la mort et à la renaissance.

\section{RÉFÉRENCES BIBLIOGRAPHIQUES:}

ARISTOTE. L'homme de génie et la mélancolie. Problème XXX. Traduit du grec par Andrea L. Carbone et Benjamin FAU. Paris: Editions Allia, 2004.

BARGENDA, Angela. La Poésie D'Anna de Noailles, Paris: L'Harmattan, 1995.

BERCOT, Martine. Anthologie de la Poésie Française: du XVIIème au XXème siècle. Paris: Gallimard, 2000.

BRUNEL, Pierre. Mythopoétique des genres. Paris: PUF, 2003.

. Dictionnaire des Mythes Littéraires. Paris: Du Rocher, 1998.

CHIANCA, Karina. L'amour en échec: lyrisme et mélancolie chez, Guillaume Apollinaire et Vinicius de Moraes. João Pessoa: Idéia, 2007.

. Métapsychologie. Traduit de l’allemand, éd. revue et corrigée par Jean Laplanche et J.- B. Pontalis. Paris: Gallimard, 1968.

HERSANT, Yves. Mélancolies: de l'Antiquité au XXème siècle. Paris: Robert Laffont, 2005.

MAULPOIX, Jean-Michel. Du lyrisme. Paris: librairie José Corti, 2000.

NOAILLES, Anna. L'ombre des Jours. Paris: Calmann-Lévy, 1902. disponible sur < http//www. gallica.bnf.fr>, consulté le 25 avril, 2008.

. Le coeur innombrable. Paris: Calmann-Lévy, 1901. disponible sur $<$ http//www.gallica.bnf. fr>, consulté le 25 avril, 2008.

PRINGENT, Michel. Histoire de la France Littéraire, Modernités XIX et XXème siècle. Paris: PUF, 2006. 